\title{
Ubiquitous System for Events Promotion
}

\author{
Emanuel Soares Peres Correia ${ }^{1}$, Nuno André Osório Liberato, Maximino \\ Esteves Correia Bessa ${ }^{1,2}$ and João Eduardo Quintela Alves de Sousa \\ Varajão ${ }^{1,3}$ \\ 1 Universidade de Trás-os-Montes e Alto Douro, 5001 Vila Real, Portugal. \\ 2 Instituto de Engenharia de Sistemas e Computadores, 4200 Porto, Portugal.
} 3 Centro ALGORITMI, 4700 Braga, Portugal.

\begin{abstract}
The potential of mobile devices has been steadily increasing with time. Accordingly, Smartphones are nowadays an almost ubiquitous platform, with great hardware and software capabilities regarding location-based applications, from which only navigation-aid systems are being thoroughly explored. However, despite such growth, e-commerce platforms are not yet totally prepared to cope with mobile devices singularities, namely screen size and input devices, which is delaying the use of mobile devices as commerce and advertising gateways to users. On the other hand, dedicated and customizable software solutions, ready to cope and even to take advantage of mobile devices characteristics, may turn out to be a simple way of placing M-commerce on the road to full user adoption and global success. As a result, this paper proposes a new e-business system architecture meant for events promotion, allowing users to search for cultural events, based on their geographic location and only by using a mobile device. In view of that, if an interesting event is found, users are therefore given further detailed information, such as text, image, sound or even small video trailers, as well as reserve or even buy tickets.
\end{abstract}

Keywords: Ubiquitous, Cultural-Events, LBMS, E-business, Mobile-Devices.

\section{Introduction}

The wide spread use of mobile devices is an already unquestionable achievement, easily confirmed by the very number of per capita devices found in several different countries or by the remarkable global penetration rate of $61 \%$ by the end of 2008 (ITU, 2008).

Although, such devices were previously made of a relatively limited set of both software and hardware related functionalities and capabilities, providing nothing but voice communication, SMS

Copyright (C) 2010 Emanuel Soares Peres Correia, Nuno André Osório Liberato, Maximino Esteves Correia Bessa and João Eduardo Quintela Alves de Sousa Varajão. This is an open access article distributed under the Creative Commons Attribution License unported 3.0, which permits unrestricted use, distribution, and reproduction in any medium, provided that original work is properly cited. Contact Author: Emanuel Soares Peres Correia, e-mail: eperes@utad.pt 
(Short Message Service) and little more. There has been a significant progress in its characteristics sphere in recent years. In fact, it has become quite ordinary to find devices equipped with a wide range of technologies, adding up to an already existent significant processing capacity, distinct communication technologies like GPRS (General Packet Radio Service), UMTS (Universal Mobile Telecommunications System), 802.11x, Bluetooth, Infrared and NFC (Near Field Communication), location abilities such as GPS (Global Position System) or through one's service provider, detection of movement using accelerometers, among others (Hernandez, 2009; Reynolds, 2009; Want, 2009).

Such assembly of technical abilities allow the equipments to support complex software applications as well as the execution of several services, including LBMS (Location-Based Mobile Services). Therefore, and despite this rich set of features and the engendering of numerous possibilities resulting from its combination, the most significant and overall advantage being exploited from the location-based abilities coincides with the navigation-aid systems (Arikawa, 2007; Seok-Won Lee and Vason P. Srini and Joo-Hyeon Lee and Young-Woo Sohn and Yoon Su Baek and Sang-Yong Lee and WooShik Kang and Seong Woon Kim, 2005), accurately meaning that mobiledevices software and hardware potential are not being fully used. On the other hand, bringing environmentcontextualized information and services to users through their mobile-devices seems yet to be roughly explored as an electronic business, despite this is quickly changing (Helal, 2008; Oriana, Veli-Matti, Sebastian, \& Lasse, 2008; VaughanNichols, 2009). Consequently, it is in the very context of the present article that we come up with the system as follows: a system that, taking advantage of the capabilities of processing, communication and location of current mobile devices, allows a given user to obtain contextaware information regarding events of her/his own interest.

Let's take the following example: when someone is on tour and spontaneously wants to find information about cultural or other kind of events that are taking place in their geographical surroundings, up to present it is not known an available system or service capable of providing it.

Thus, so far, in order to obtain this information, the immediate solution might be to use an unstructured search mechanism of information, for example:

- Research in thematic portals on the Internet, though restricted to the extent of the type adapted to smaller screens of mobile devices. Besides information is not filtered against the search as selected, interfaces are not simple because they are designed for desktop use, nor optimized for use on mobile devices;

- Approach an information spot (a touristic post, for instance), however with limitations concerning geographical localization and respective timetable availability and also not possessing all events;

- Ask a passer-by, which involves limitations, such as dependence on knowledge of the passer-by, language barriers or unwillingness to provide the requested information.

As a consequence, these solutions share several problems, namely: the unstructured information, the ease of access is not pre-determined, incomplete and rather poor information; limited possibilities (for example, asking somebody if there is a theater event nearby does not allow to book tickets). 
To overcome this problem, we propose a new ubiquitous events promotion ebusiness system, which enables one to search for cultural events based on geographic location, also supporting ticket-booking and ticket-selling services. The system consists of several components whose worthy of mention are the following: an application for mobile devices, a repository of cultural events and a gateway designed for tickets reservation. It is intended that a user, by means of both his mobile device and an application previously installed, may be able to ask a repository of cultural events which activities are or will be presented, or even try an activity that interests him/her in particular, for example, using the name of a film as a search keyword. The repository will indeed return the user the results regarding his/her request, properly georeferenced. For such purpose, and assuring the user's automatic and transparent conveniences, the application informs the system about his/her location and possible preferences (for example, the user may prefer cultural activities in a given interval of time). Consequently, the user will be granted several functionalities, such as watch multimedia content regarding the event, book or buy tickets and, furthermore, validate tickets.

This paper is structured as follows: the next section presents the literature review, followed by the presentation and discussion of the system architecture and then the analysis of the system prototype. The final section is completed with some relevant conclusions obtained from this paper.

\section{Background}

The ever growing and wide spread of mobile devices with increasing technological capacity playing the role of mobile computing platform together with a particular feature that stays permanently close to the personal life of individuals (Raento, Oulasvirta, Petit, \& Toivonen, 2005; Srivastava, 2005), explains its status as privileged tools for the development and implementation of the concept "context-aware" as applied to applications and services (Raento et al., 2005; Rao \& Minakakis, 2003). Currently, it is, in fact, the real-time obtained geographical location the preferred basis upon the services with context related information, in which the individual is also included, are provided (Toye, Sharp, Madhavapeddy, \& Scott, 2005). Obtaining this information has been facilitated and has become more efficient, besides fast, mainly due to the widespread introduction of technologies such as GPS in mobile devices or the use of networks of service providers (Rao \& Minakakis, 2003).

The concept of LBMS opens in a new direction to the development of content and applications for mobile platforms (Helal, 2008; Vaughan-Nichols, 2009), as well as ways to integrate the latter concerning its hardware particularities and communications - into the already existent and working digital services networks, presenting, however, the opportunity to innovate because an ubiquitous and personal vehicle together with growing multimedia facilities, are capable of leading to real-time relevant, personalized and contextualized services and contents, which can be presented transparently to the user and in the very palm of his/her hand (Toye et al, 2005).

With regard to the economic potential that may be well acquired by service providers, their profits range from traditional operators to virtually any entity with a web platform ready to be accessed by mobile devices or to provide their services using web services (Farley \& Capp, 2005; Oriana et al., 2008). This stands for a whole new market that 
combines together the mobility and contextual domain, a role very much played by the ever present element of dayto-day: the mobile phone. There are already some examples of location-based services for individuals, namely services of virtual waiting lines, issuing tickets for transport, social networks, and others, being then briefly described as an example.

A service of virtual waiting lines is described in (Toye et al, 2005), enabling a customer of a restaurant need not be expected to hold a table in the lobby. Using a mechanism of local context - a visual tag (QR Code) the client uses his/her mobile device to photograph the element of context and an application previously installed to decode the content, which in this case contains a series of addresses providing Bluetooth connections between the client's mobile device and restaurant's server. The customer is, therefore, informed about the average time of waiting, the number of people standing ahead of oneself and then asked whether if one desires or not to be placed in the virtual queue. If yes, the customer is then asked to indicate how many people will be served when available. Finally, the client may now wait for his/her turn lightly, taking time to go shopping or doing any other kind of service that he/she is required to accomplish. The user will then be notified by the system of the restaurant, through SMS, letting client know about when to go to and be served in the shortest time possible.

Additionally, the SitOrSquat.com (SitOrSquat.com, 2009) site puts forward the possibility to find, for example, a bathroom near your present setting. In detail, as soon as we insert a given location within the map as presented it moves towards the selected location where we may find all the bathrooms registered in the systems which are thus shown.
Furthermore, when we click on the bathroom icon, a small window containing basic information about that bathroom appears and if we click on the icon corresponding to the name of the bathroom more and detailed information on another window is also made available. It is again possible to use this service on mobile phone by downloading it to your Blackberry or iPhone. It is also possible to find precisely how to get to the nearest bathroom by again sending a SMS and following the instructions as provided.

The WhosHere (Honan, 2009) consists of an application set for digital social networks and is also able to show other users that are geographically close, through the basis of provided information about the current location of the individual, and likewise facilitate the interaction between them by means of short written messages. Similarly, it also allows performing searching activities among available users, based on criteria such as whether the person is available or not for friendship relationships, casual encounters, among others. Furthermore, accessing users' profiles and further exchange of their respective multimedia information, like photographs, turned out to be as well possible.

The Mobile Cab Finder, CAB4Me (cab4me.com, 2009), consists of an application whose main feature relies in making easier for us to find a taxi and is available for the T-Mobile G1 Android phone. All we have to do is to choose our location/setting on a map and we are then shown where the nearest taxi is, if available. By clicking on the call tab the local cab companies are likewise illustrated. If registered on the database, the companies and its related information, such as payment methods and car types, are also on hand. If there are no registered cabs for one area, a local web search is performed. 
Selling public transports tickets based on the user location (Bohm, Murtz, Sommer, \& Wermuth, 2005) is a relatively new reality coming with a really simple concept: the user dials a check-in number when one is about to start using public transports and is located using their mobile services provider and multimedia activities, such as photographs, among them. Next, and once one takes the public transport and selects the journey, within the area covered by the service, it goes all the way through and as long as one desires. It only takes the user a quick contact to the check-out number when the journey is finished. Thus, based on the initial position, final position and the public transports network, the service is able to calculate the value to charge and follows by automatically deducting it from the account belonging to client's mobile operator. Finally, tickets selling for sport events and music concerts, as well as related promotions is also described in (Farley \& Capp, 2005).

\section{System architecture}

The management system of events that we propose relies on the availability of information and services, based on the location of those who request them. Consequently, it is intended that a given user by means of a mobile device with a previously installed application, may be allowed to request a repository of cultural events, information on activities that are or will be taking place nearby or even try something that he/she likes in particular, such as a film. The referred repository will indeed return the user and properly geo-reference results corresponding to his/her request. For such purpose, the application installed on the mobile device informs the repository system, in a transparent way to the user, about his/her location and possible preferences (for example, the user may well prefer cultural activities in a given time frame). The user will be then presented with a series of events, within which a selection can be made, according to his/her interests (eg, multimedia content, such as trailers). The user is also able to make a reservation or purchase tickets for events, in which case will be provided directly in his/her device ready to be taken to the site of the event and be later object of automatic validation.

As can be seen in Figure 1, the system Location-Based Event Promoter Service (LBEPS) is made of several components that are described below: Mobile Device (MD), Events Location Based Search Engine (ELBSE); Events Promoters (EP); Events Organizers (EO); Multimedia Repositories (MMR); Ticket Gateway (TG); Ticket Control Devices (TCD). 


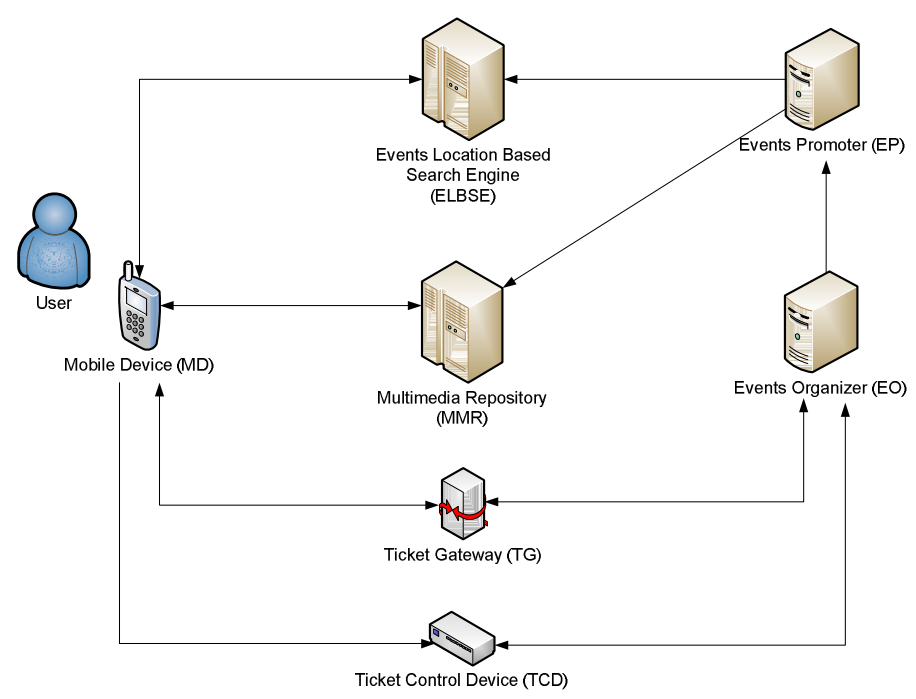

Figure 1 - LBEPS Global Arquitecture

MD: It stands for devices such as a PDA or mobile phone which enable the user to search for events in a selected geographical area, give access to multimedia information like videos or photos, purchase tickets for several events and finally validate the ticket on the location where the events take place.

E0: This system is hold by an entity that manages proceedings such as theatre events. These are the exact systems that register several different type of activities such as the events (i.e. latest James Bond film), the related multimedia promotion material (i.e. trailer) concerning the event and the tickets information.

EP: It is an entity owned by a promoter of events (e.g. a promotion agency), which is responsible for the registration of events (e.g. performances) in ELBSE and recording of multimedia promotional material in MMR

ELBSE: It is a search engine that allows both the EP and EO systems, in a primary and initial stage of the process, register the events, and later, in a second phase, reply the requests on events search by MD.

MMR: It is a server system that holds record of the multimedia promotional material regarding events.

TG: It represents a system thought of to provide services concerned with both reservation and purchase of tickets and acts as the interface between EO and MD.

TCD: This device is designed to control the users entrance/access to events. The user is then submitted to an authenticated verification based on the information retrieved by the mobile device, finally validating the request on the EO.

In the overall operation of the system, it is possible to identify several of its moments, whose messages exchanged among the various components are represented in Figure 2 as follows: 




Figure 2 - LBEPS architecture detail (general exchanged messages)

First moment:

- Each EO system is registered in EP system;

- Each EO system is registered in TG system;

- Each EP system is registered in ELBSE system;

- Each EP system is registered in MMR system.

Second moment:

- Each event is recorded in EO system;

- By EO system, the events are recorded in TG systems (message "Register event") and EP (message "Register event");

- By EP system, events are recorded in the ELBSE system (message "Submit event");

- By EP system, the multimedia elements of the events are recorded in the MMR system (message "Submit media").

At the final stage of the second moment the running events will become available in the ELBSE system for further search.

Third moment:
- The user, by employing an MD, configures the system by introducing some parameters, such as the aspects that interest him/her the most (e.g. a music concert, theater performance) and the maximum distance from a specific location;

- Meanwhile, at that moment, it is sent the "Search event" message from the MD system to the ELBSE one, indicating what search is wanted. Through the latter it is accordingly sent a response by the ELBSE to the MD system, containing the list of events resulting from the search as previously conducted;

- If the user wishes to access multimedia elements of a given event he may confer the list of events presented after finishing the search. After selecting a certain event, a "get media" message will be sent from the MD system to the EO system and/or the EP system, depending on where the multimedia elements are stored up. Once the request is assured the user may 
at last visualize the available multimedia elements;

- If the user is longing to purchase tickets for a certain event, he may do it by selecting the elected event followed by the indication of the number of tickets he would like to pay for. For which purpose it will be sent a "buy tickets" message from the MD system to the EO system. As a feedback, if tickets are still available, the user will be delivered an electronic ticket through his MD.

Fourth moment:

It is when the user sets off to the event where he/she will make use of his/her MD in order to get the electronic tickets authenticated. At this moment a "validate ticket" message will be sent from the MD system to the TCD system, which, in its turn, dispatches a "validate ticket" message to the EO system. As soon as the ticket is considered valid the user will indeed get the signal allowing him/her to get into the place where the event he previously selected is taking place.

\section{Prototype}

The LBEPS system was put into practice by making use of the Android operating system for mobile devices exclusive application purposes, whereas Linux proved relevant in the system repository of events. Such performance did implicate the use of a range of technologies, such as Java, Web services (PHP), Apache and MySQL. In general, these technologies were used because they are open source, which reduces the costs of implementing and are furthermore supported by a broad community of users, which enhances its development with the steady addition of new features

Regarding the recent operating system named Android, enabling nonetheless a direct interaction with the Google Apps (e.g. Google Maps), it has got significant advantages in developing applications in a well established language (JAVA), not to mention the remarkably ever growing applications market developed by its supporting community.

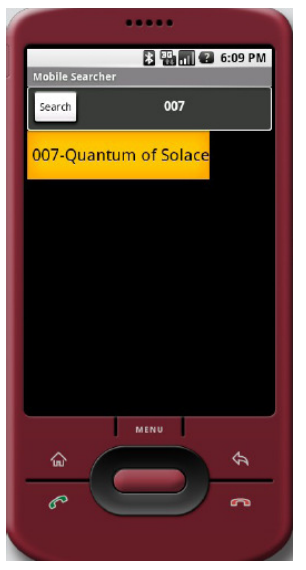

Screenshot 3

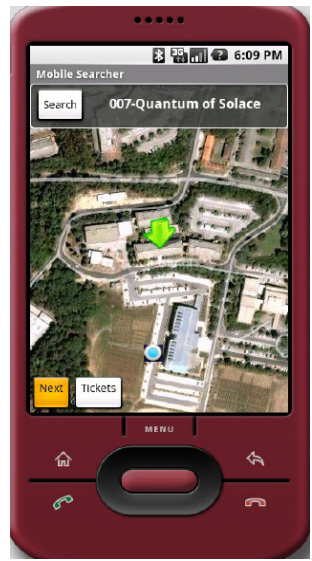

Screenshot 4

Figure 3 - LBEPS prototype screenshots 
In Figure 3 there are several screenshots of the application especially designed for mobile devices.

When started, the application automatically loads a global terrestrial map indicating the present location of the user and providing him/her the possibility to start a given search (screenshot 1, "Search" option). Once the map is loaded, the user is able to navigate freely on it, as well as zoom in, zoom out and change the way the map is showed. Once the "Search" option is selected, a small window shows up in the middle of the screen to allow the user to insert the search keywords dealing with the event he/she wants to look for (screenshot 2).

In case the user does not have any defined preference to fill in the corresponding field, he/she may still carry out the research leaving it unfilled. The confirmation of the undergoing research (screenshot 2, button "ok"), will activate an HTTP request to be sent to a web service. The HTTP request will therefore contain a Simple Object Access Protocol (SOAP) message with several parameters: the event introduced the server address and the function that will be called. Subsequently, the web service returns all the events containing the word introduced and the results are shown on a new window (screenshot 3). To decide what event is to be shown, the user must select an event from the list by clicking on it, minding that if the event is for some reason not listed he/she can, however, click on the return button and go back again to the previously map window and insert another event.

Once the selected event is retrieved, the user is then exposed to the map exactly indicating the location of the event nearby her/him. As future work and consequent developments the prototype features are expected to put into action the reservation/purchase of electronic tickets facilities and its later validation, every time the user sets off to participate in the events she/he chooses.

\section{Conclusions}

LBMS will quickly grow to become part of everyday routines, mainly because they offer contextualized real-time services which will help in professional and personal life, through one's own everpresent mobile device.

Hence, why did not the LBMS become a hit yet? We believe that what is still keeping these services in labs is the lack of a rich experience undertaken by the user. In fact, the first generation of LBMS provided the user nothing but contextualized information, whose services would, for example, provide information about available theatres in certain surroundings to take the example previously presented. However, its limits are such to the extent of not being able to offer the user further details as the number and names of each film on screen, the possibility of buying a ticket for the James Bond latest film, the shortcut, if any, can the user take to go to the theatre. These exemplified tasks define the very rich experience that may be granted to users if they adopt these 2.0 LBMS.

The system as proposed in this article comprises a whole new way through which users may indeed search and obtain information about events taking place in their geographical proximity, providing an effective interface skilled at obtaining structured, focused and timely information, which, so far up to present, is not possible to achieve by any other means with similar efficiency. Therefore, we strongly believe and support that the system we have been studying and describing represents a contribution and a new step in the context of LBMS systems. 


\section{References:}

Arikawa, M. K., S. Ohnishi, K. (2007). Navitime: Supporting Pedestrian Navigation in the Real World. Pervasive Computing, IEEE, 6(3), 21-29.

Bohm, A., Murtz, B., Sommer, G., \& Wermuth, M. (2005). Location-based ticketing in public transport. Paper presented at the Intelligent Transportation Systems, IEEE.

cab4me.com. (2009). Mobile Device Application. from http://beta.cab4me.com/orderman/index.ht $\underline{\mathrm{ml}}$

Farley, P., \& Capp, M. (2005). Mobile Web Services. BT Technology Journal, 23(3), 202213.

Helal, P. B. a. A. K. a. S. (2008). LocationBased Services: Back to the Future. IEEE Pervasive Computing, 7(2), 85-89.

Hernandez, E. A. (2009). War of the Mobile Browsers. IEEE Pervasive Computing, 8(1), 82-85.

Honan, M. (2009, 17/02). I Am Here: One Man's Experiment With the Location-Aware Lifestyle. Wired Magazine.

ITU. (2008). Worldwide mobile cellular subscribers to reach 4 billion mark late 2008. from http://www.itu.int/newsroom/press releas es/2008/29.html

Oriana, R., Veli-Matti, T., Sebastian, S., \& Lasse, H. (2008). A Next Generation Operator Environment to Turn ContextAware Services into a Commercial Reality, Proceedings of the The Ninth International Conference on Mobile Data Management \%@ 978-0-7695-3154-0 (pp. 90-97): IEEE Computer Society.
Raento, M., Oulasvirta, A., Petit, R., \& Toivonen, H. (2005). ContextPhone: a prototyping platform for context-aware mobile applications. Pervasive Computing, IEEE, 4, 51-59.

Rao, B., \& Minakakis, L. (2003). Evolution of mobile location-based services. Commun ACM, 46(12), 61-65.

Reynolds, F. (2009). Web 2.0-In Your Hand. IEEE Pervasive Computing, 8(1), 86-88.

Seok-Won Lee and Vason P. Srini and JooHyeon Lee and Young-Woo Sohn and Yoon Su Baek and Sang-Yong Lee and WooShik Kang and Seong Woon Kim, T.-D. H. a. C. C. a. H.-M. Y. a. J.-Y. K. a. S.-H. J. a. Y.-S. R. a. B.-S. K. a. H.-K. K. a. (2005). Implementation of New Services to Support Ubiquitous Computing for Town Life. Software Technologies for Future Embedded and Ubiquitous Systems, IEEE Workshop on, 0, 45-49.

SitOrSquat.com. (2009). Web and Mobile Device Application. from http://www.sitorsquat.com/sitorsquat/ho $\underline{\text { me }}$

Srivastava, L. (2005). Mobile phones and the evolution of social behaviour. Behaviour and Information Technology, 24(2), 111-129.

Toye, E., Sharp, R., Madhavapeddy, A., \& Scott, D. (2005). Using smart phones to access site-specific services. Pervasive Computing, IEEE, 4(2), 60-66.

Vaughan-Nichols, S. J. (2009). Will Mobile Computing's Future Be Location, Location, Location? Computer, 42(2), 14-17.

Want, R. (2009). When Cell Phones Become Computers. IEEE Pervasive Computing, 8(2), 2-5. 